\title{
An Improved Method for Using Mg in to Estimate Black Hole Masses in Active Galactic Nuclei
}

\author{
Christopher A. Onken ${ }^{1,2} \&$ Juna A. Kollmeier ${ }^{3}$ \\ onken@mso.anu.edu.au, jak@ociw.edu
}

\begin{abstract}
We present a method for obtaining accurate black hole $(\mathrm{BH})$ mass estimates from the Mg II emission line in active galactic nuclei (AGNs). Employing the large database of AGN measurements from the Sloan Digital Sky Survey (SDSS) presented by Shen et al., we find that AGNs in the redshift range 0.3-0.9, for which a given object can have both $\mathrm{H} \beta$ and $\mathrm{Mg}$ II line widths measured, display a modest but correctable discrepancy in Mg II-based masses that correlates with the Eddington ratio. We use the SDSS database to estimate the probability distribution of the true (i.e., $\mathrm{H} \beta$-based) mass given a measured $\mathrm{Mg}$ II line width. These probability distributions are then applied to the SDSS measurements from Shen et al. across the entire Mg II-accessible redshift range (0.3-2.2). We find that accounting for this residual correlation generally increases the dispersion of Eddington ratios by a small factor $(\sim 0.09$ dex for the redshift and luminosity bins we consider). We continue to find that the intrinsic distribution of Eddington ratios for luminous AGNs is extremely narrow, 0.3-0.4 dex, as demonstrated by Kollmeier et al. Using the method we describe, Mg II emission lines can be used with confidence to obtain $\mathrm{BH}$ mass estimates.
\end{abstract}

Subject headings: quasars: emission lines

\footnotetext{
${ }^{1}$ Dominion Astrophysical Observatory, Herzberg Institute of Astrophysics, National Research Council of Canada, 5071 West Saanich Road, Victoria, BC, V9E 2E7, Canada

${ }^{2}$ Current address: Research School of Astronomy and Astrophysics, The Australian National University, Cotter Road, Weston Creek, ACT 2611, Australia

${ }^{3}$ Observatories of the Carnegie Institution of Washington, 813 Santa Barbara Street, Pasadena,
}

\section{INTRODUCTION}

The $M_{\mathrm{BH}^{-}} \sigma$ correlation between supermassive black hole $(\mathrm{BH})$ mass and the velocity dispersion of the surrounding stellar system indicates a significant connection between galaxy and $\mathrm{BH}$ assembly (Ferrarese \& Merritt 2000; Gebhardt et al.

CA 91101 
2000). Thus, it is essential to establish the most basic $\mathrm{BH}$ parameters: their intrinsic distribution of masses and growth rates.

It is extraordinarily difficult to measure BH masses directly, even in the nearby universe, because of the small spatial scales that must be resolved to probe the gravitational influence of the $\mathrm{BH}$. In active galactic nuclei (AGNs), the technique of reverberation mapping (Blandford \& McKee 1982; Peterson 1993) employs high resolution in the time domain to probe gas dynamics on spatial scales close to the $\mathrm{BH}$. However, the long-duration spectroscopic monitoring campaigns required for reverberation studies currently preclude the method from being applied to large numbers of objects. Therefore, one must rely on even more indirect techniques of BH mass estimation to build up statistically significant samples.

The "virial" method has been empirically calibrated from reverberation mapping experiments (Wandel et al. 1999; Vestergaard 2002; McLure \& Jarvis 2002) and allows a $\mathrm{BH}$ mass estimate from a one-time measurement of the width of a broad emission line and the AGN luminosity (see $\S 2$ ). To facilitate application of the virial technique to large optical AGN surveys, versions have been developed for $\mathrm{H} \beta$ at low redshift, for the $\mathrm{Mg}$ II doublet near $2800 \AA$ at intermediate redshift, and for the $\mathrm{C}$ IV doublet near $1550 \AA$ at high redshift (for recent prescriptions, see Vestergaard \& Peterson 2006; McGill et al. 2008).

In this paper, we present evidence of a systematic discrepancy in Mg II-based BH mass estimates as a function of Eddington ratio (the ratio of the bolometric luminos- ity, $L_{\text {bol }}$, to the luminosity required for radiation pressure to balance the gravity of the $\mathrm{BH}$ ), as well as a method to correct this trend.

\section{Method of Analysis}

The standard equation for estimating $\mathrm{BH}$ masses in AGNs from single-epoch spectroscopy is:

$$
\log M_{\mathrm{BH}}=a+b \log L_{c}+2 \log V,
$$

where $V$ is the width of the broad emission line, $L_{c}$ is the continuum luminosity near the line, and $a$ and $b$ are constants (which vary from line to line). The dependence on $L_{c}$ arises because the distance of the broad emission line gas from the $\mathrm{BH}$ has been observed to correlate tightly with the AGN luminosity over 4 orders of magnitude in $L_{c}$ (Bentz et al. 2007). Thus, the mass equation reverts to the simple, virial combination of radius and velocity. The calibration of these relations rests on the bedrock of reverberation mapping measurements of local AGNs. By far, the best reverberation mapping dataset exists for $\mathrm{H} \beta$ (Peterson et al. 2004). The $a$ and $b$ coefficients for both $\mathrm{C}$ IV and Mg II principally rely on empirical correlations with $\mathrm{H} \beta$ reverberation masses. However, whereas C IV reverberation studies of a handful of objects are consistent with expectations from $\mathrm{H} \beta$ (Peterson et al. 2005), a clear indication of reverberation has not yet been found for Mg II. Although the line has been seen to vary in both flux and width (e.g., Clavel et al. 1991; Dietrich \& Kollatschny 1995; Metzroth et al. 2006; Woo 2008), only weak $\mathrm{Mg}$ II reverberation signals have been seen (Reichert et al. 1994). Thus, the mass relation for $\mathrm{Mg}$ II relies on the 
correlation of single-epoch estimates with $\mathrm{H} \beta$ masses and the argument that $\mathrm{Mg}$ II and $\mathrm{H} \beta$ have similar ionization potentials (McLure \& Dunlop 2004).

With the wide wavelength coverage of Sloan Digital Sky Survey (SDSS) spectra, certain redshift windows allow for two of these three emission lines to be measured simultaneously: $\mathrm{H} \beta$ and $\mathrm{Mg}$ II are both accessible for $z \sim 0.3-0.9$, while $\mathrm{Mg}$ II and C IV can both be measured for $z \sim 1$.72.2. By comparing the $\mathrm{BH}$ mass estimates from the two lines, we are thus able to study systematic trends. Such a comparison has been done previously for the mean relation, but the large sample size of the SDSS permits an analysis of higher-order correlations, which prove to be quite important.

Shen et al. (2008) have provided line width (FWHM) measurements and $\mathrm{BH}$ mass estimates - using the relations of McLure \& Dunlop (2004) and Vestergaard \& Peterson (2006)-for roughly 60,000 AGNs from the SDSS, including $\sim 8,000$ with both $\mathrm{H} \beta$ and $\mathrm{Mg}$ II, and $\sim 15,000$ with both Mg II and C IV. They provide a detailed analysis of the relationship between Mg II and C IV, but simply describe the ratio of $\mathrm{H} \beta$ to $\mathrm{Mg}$ II FWHM as following a log-normal distribution with a mean of 0.0062 dex and a dispersion of 0.11 dex. Shen et al. noted that the relation of the two FWHMs deviates slightly from a perfect correlation, but did not explore the issue further.

Under the premise that $\mathrm{H} \beta$, being the most extensively reverberation-mapped emission line, provides the best indicator of the $\mathrm{BH}$ mass, we examine AGNs having both $\mathrm{H} \beta$ and $\mathrm{Mg}$ II mass measurements.
Due to uncertainties in the line width measurements, we exclude the small number of AGNs that were flagged by Shen et al. as broad absorption line objects. However, the inclusion of these objects has negligible effect on our results. In Figure 1, we plot the difference in the (log of the) $\mathrm{BH}$ mass as a function of Eddington ratid 1 . The strong correlation implies that if $\mathrm{Mg}$ II is calibrated simply from the mean of the $\mathrm{H} \beta-\mathrm{Mg}$ II relation, then it will underestimate the $\mathrm{BH}$ mass at low Eddington ratio and overestimate the mass at high Eddington ratid2. This would lead one to infer a narrower distribution of Eddington ratios than actually exists. One way to quantify this effect is to measure the slope of the correlation

$$
\beta=\tan \left[\frac{1}{2} \arctan \frac{2 c_{12}}{c_{11}-c_{22}}\right]
$$

where $c_{i j}$ is the covariance matrix of the distribution shown in Figure 1, A slope $\beta=0$ would imply that $\mathrm{Mg}$ II provided completely independent information on the $\mathrm{BH}$ mass, while $\beta=1$ would imply that no information is conveyed by the $\mathrm{Mg}$ II measurement. The actual value is $\beta=0.76$, which means that Mg II is indicative of the BH mass, but must be treated with care.

Simply modifying the luminosity-dependence of the $\mathrm{Mg}$ II mass formula (i.e., changing $b$ in eq. [1]) cannot remove the observed trend. Therefore, to make a statistical correction to the Mg II-based masses, we adopt the following method. For the SDSS

\footnotetext{
${ }^{1}$ The Eddington ratio is computed with the $\mathrm{H} \beta$ based BH mass.

${ }^{2}$ We note that the same trend from Figure 1 is seen when replacing FWHM with the inter-percentile value measurements of Fine et al. (2008).
} 
objects with both $\mathrm{H} \beta$ and Mg II FWHMs, we look at AGNs with $\mathrm{Mg}$ II lines in a given 0.1 dex bin of FWHM and tabulate the distribution of $\mathrm{H} \beta$ FWHMs for those objects. We take those $\mathrm{H} \beta$ distributions (normalized appropriately) as the probability distributions for the true FWHM underlying the observed Mg II value (Fig. 2; Table 1).

For any object with an accessible $\mathrm{Mg}$ II line, each bin in true FWHM is combined with the observed continuum luminosity to calculate a $\mathrm{BH}$ mass (via eq. [1]), and the mass is then used with the object's bolometric luminosity to derive an Eddington ratio. The probability for each bin of FWHM is added to the total number of objects contributing to the corresponding Eddington ratio bin (which are 0.2 dexwide, allowing a one-to-one match between FWHM and Eddington ratio bins). Thus, each Mg II FWHM becomes a weighted distribution of Eddington ratio $\$ 3$, while $\mathrm{H} \beta$ - and $\mathrm{C}$ IV-based Eddington ratios contribute directly at their observed values.

We now apply this technique to the Shen et al. measurements of the uniformly selected subsample of SDSS AGNs (Richards et al. 2006).

\section{Results \& Discussion}

We construct distributions of Eddington ratios for SDSS in ranges of $\left(L_{\mathrm{bol}}, z\right)$ in Figure 3, showing the results both with (solid) and without (dotted) the Mg II sub-

\footnotetext{
${ }^{3}$ If the observed $\mathrm{Mg}$ II FWHM falls in a bin in which there were no $\mathrm{H} \beta+\mathrm{Mg}$ II measurements, it is given a probability of 1 within the bin corresponding to the Mg II FWHM. In the sample we consider, this applies to a single AGN.
}

stitution presented above. The statistics of the distributions are given in Table 2 . After correcting the Mg II measurements in the uniformly selected SDSS sample, we find that the average width of the Eddington ratio distribution increased by 0.09 dex for objects in the redshift range 0.3-2.2. We therefore find that the distribution of Eddington ratios remains very narrow at $\sim 0.4$ dex, as was found by Kollmeier et al. (2006) for the AGES-I survey.

To test our procedure, we apply it to multiple subsamples for which we have both $\mathrm{H} \beta$ and $\mathrm{Mg}$ II data. Figure 4 shows the distribution of Eddington ratios for each subsample calculated in two ways, first using the true $\mathrm{H} \beta$ mass (solid) and second using our procedure applied to the Mg II mass (dashed). The similarity of these distributions (and the differences from the raw Mg II-based values, shown as the dotted histograms) demonstrates that our procedure works well, recovering the true Eddington ratio distribution from the $\mathrm{Mg}$ II derived masses.

The general trend we observe could be explained physically if the location where lines are formed in the broad-line region depends on accretion rate. In this case, the radius-luminosity relation would also depend on Eddington ratio and that would introduce an additional term in the virial mass relation. It would then be possible, in principle, to remove this dependence entirely analytically by fitting the observed correlation. We investigate this further in an upcoming work.

The virial method for BH mass estimation has opened a new window in the study of supermassive $\mathrm{BH}$ demographics. While each indicator has systematics that must 
be addressed (i.e., asymmetric line profiles, contamination from disk winds and metal lines, etc.), it is important to understand and attempt to correct for these systematics so that this technique can be applied with confidence. In this contribution, we have identified a limitation in estimating $\mathrm{BH}$ masses from the $\mathrm{Mg}$ II line and presented a way to remove it by exploiting the overlap of $\mathrm{Mg}$ II and $\mathrm{H} \beta$ measurements presented by Shen et al. (2008). This method puts Mg II masses more securely on the same scale as $\mathrm{H} \beta$, which should be the most reliable in these studies. As the bias in the Mg II masses is also seen in our analysis of the 2dF Quasar Redshift Survey (2QZ) and the AGN and Galaxy Evolution Survey (AGES), in forthcoming work, we will apply our correction technique to those datasets (including the expanded sample of AGES-II).

Understanding the transition between high-luminosity, high-redshift AGNs having a narrow Eddington ratio distribution ( $\sim 0.4 \mathrm{dex})$ and low-luminosity, lowredshift AGNs with a broad Eddington ratio distribution (>1 dex; Ho 2002; Woo \& Urry 2002) can provide important constraints on the physics of $\mathrm{BH}$ accretion. To determine these distributions, it is critical to continue to improve $\mathrm{BH}$ mass estimates that can be used through the bulk of the cosmic AGN activity.

We thank the anonymous referee for providing useful feedback. We thank Andy Gould for helpful discussions and a careful reading of an early draft of this letter. We thank Chris Kochanek for insightful comments on this manuscript. We thank Matthias Dietrich for valuable sug- gestions, and Pat Hall and Alireza Rafiee for stimulating discussions. CAO acknowledges support by a Plaskett Fellowship during the completion of this work. JAK acknowledges the support of Hubble Fellowship HF-01197 and a CarnegiePrinceton fellowship during the completion of this project. This research was supported in part by the National Science Foundation under Grant No. PHY0551164. Funding for the SDSS and SDSSII has been provided by the Alfred P. Sloan Foundation, the Participating Institutions, the National Science Foundation, the U.S. Department of Energy, the National Aeronautics and Space Administration, the Japanese Monbukagakusho, the Max Planck Society, and the Higher Education Funding Council for England. The SDSS Web Site is http://www.sdss.org/.

\section{REFERENCES}

Bentz, M. C., Denney, K. D., Peterson, B. M., \& Pogge, R. W. 2007, in ASP Conf. Ser. 373: The Central Engine of Active Galactic Nuclei, eds. L. C. Ho \& J.-M. Wang (San Francisco: ASP), 380 (astro-ph/0702650 v1)

Blandford R. D., \& McKee C. F. 1982, ApJ, 255, 419

Clavel, J., Reichert, G. A., Alloin, D., Crenshaw, D. M., Kriss, G., \& Krolik, J. H. 1991, ApJ, 366, 64

Dietrich, M., \& Kollatschny, W. 1995, A\&A, 303, 405

Ferrarese, L., \& Merritt, D. 2000, ApJ, 539, L9 
Fine, S., et al. 2008, MNRAS, in press (arXiv:0807.1155v1)

Gebhardt, K., et al. 2000, ApJ, 539, L13

Ho, L. C. 2002, ApJ, 564, 120

Kollmeier, J. A., et al. 2006, ApJ, 648, 128

McGill, K. L., Woo, J.-H., Treu, T., \& Malkan, M. A. 2008, ApJ, 673, 703

McLure, R. J., \& Jarvis, M. J. 2002, MNRAS, 337, 109

McLure, R. J., \& Dunlop, J. S. 2004, MNRAS, 352, 1390

Metzroth, K. G., Onken, C. A., \& Peterson, B. M. 2006, ApJ, 647, 901

Peterson, B. M. 1993, PASP, 105, 247

Peterson, B. M., et al. 2004, ApJ, 613, 682

Peterson, B. M., et al. 2005, ApJ, 632, 799

Reichert, G. A., et al. 1994, ApJ, 425, 582

Richards, G. T., et al. 2006, AJ, 131, 2766

Shen, Y., Greene, J. E., Strauss, M. A., Richards, G. T., \& Schneider, D. P. 2008, ApJ, 680, 169

Vestergaard, M. 2002, ApJ, 571, 733

Vestergaard, M., \& Peterson, B. M. 2006, ApJ, 641, 689

Wandel, A., Peterson, B. M., \& Malkan, M. A. 1999, ApJ, 526, 579

Woo, J.-H. 2008, AJ, 135, 1849

Woo, J.-H., \& Urry, C. M. 2002, ApJ, 579, 530

This 2-column preprint was prepared with the AAS IATEX macros v5.2. 
TABLE 1

Mg II Replacement Matrix

\begin{tabular}{|c|c|c|c|c|c|c|c|c|c|c|c|c|c|c|c|}
\hline \multirow[b]{2}{*}{ MgII FWHM } & \multicolumn{15}{|c|}{ Probability } \\
\hline & 3.05 & 3.15 & 3.25 & 3.35 & 3.45 & 3.55 & 3.65 & 3.75 & 3.85 & 3.95 & 4.05 & 4.15 & 4.25 & 4.35 & 4.45 \\
\hline 3.05 & 1.0000 & 0.0000 & 0.0000 & 0.0000 & 0.0000 & 0.0000 & 0.0000 & 0.0000 & 0.0000 & 0.0000 & 0.0000 & 0.0000 & 0.0000 & 0.0000 & 0.0000 \\
\hline 3.15 & 0.0000 & 1.0000 & 0.0000 & 0.0000 & 0.0000 & 0.0000 & 0.0000 & 0.0000 & 0.0000 & 0.0000 & 0.0000 & 0.0000 & 0.0000 & 0.0000 & 0.0000 \\
\hline 3.25 & 0.0000 & 0.0000 & 1.0000 & 0.0000 & 0.0000 & 0.0000 & 0.0000 & 0.0000 & 0.0000 & 0.0000 & 0.0000 & 0.0000 & 0.0000 & 0.0000 & 0.0000 \\
\hline 3.35 & 0.0000 & 0.0361 & 0.0723 & 0.0964 & 0.3494 & 0.2410 & 0.0602 & 0.0602 & 0.0482 & 0.0000 & 0.0120 & 0.0120 & 0.0120 & 0.0000 & 0.0000 \\
\hline 3.45 & 0.0000 & 0.0038 & 0.0379 & 0.1061 & 0.2386 & 0.3182 & 0.1667 & 0.0530 & 0.0303 & 0.0227 & 0.0152 & 0.0076 & 0.0000 & 0.0000 & 0.0000 \\
\hline 3.55 & 0.0000 & 0.0009 & 0.0196 & 0.0559 & 0.1985 & 0.3150 & 0.2591 & 0.0801 & 0.0270 & 0.0261 & 0.0121 & 0.0028 & 0.0009 & 0.0000 & 0.0019 \\
\hline 3.65 & 0.0000 & 0.0000 & 0.0050 & 0.0153 & 0.0761 & 0.2413 & 0.3625 & 0.2061 & 0.0532 & 0.0264 & 0.0096 & 0.0023 & 0.0023 & 0.0000 & 0.0000 \\
\hline 3.75 & 0.0000 & 0.0008 & 0.0021 & 0.0076 & 0.0239 & 0.0877 & 0.2244 & 0.3410 & 0.2072 & 0.0751 & 0.0227 & 0.0046 & 0.0021 & 0.0008 & 0.0000 \\
\hline 3.85 & 0.0000 & 0.0007 & 0.0022 & 0.0126 & 0.0119 & 0.0491 & 0.0908 & 0.1555 & 0.2924 & 0.2619 & 0.1057 & 0.0134 & 0.0037 & 0.0000 & 0.0000 \\
\hline 3.95 & 0.0000 & 0.0000 & 0.0000 & 0.0000 & 0.0000 & 0.0000 & 0.0000 & 0.0000 & 0.0000 & 1.0000 & 0.0000 & & 0.0000 & 0.0000 & 0.0000 \\
\hline 4.05 & 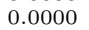 & 0.0000 & 0.000 & 0.0412 & 0.0619 & 0.1031 & 0.1340 & 0.1340 & & 0.1237 & & & & & 0.0000 \\
\hline 4.1 & & 0 & & 0.0 & 0.1 & & 0.0 & 0.8 & & & & & & & 0.0000 \\
\hline 4.2 & & 0 & 0 & 0 & & 3 & 0.3 & & & & & & & & 0.0000 \\
\hline 4.3 & & & & & & & & & & & & & & & 000 \\
\hline 4.4 & 0000 & 0 & 0 & 0 & 0 & 0.0000 & 0.0000 & 0.0000 & 0.0000 & 00 & 0.0000 & 0.0000 & 0.0000 & 0.0000 & 1.0000 \\
\hline
\end{tabular}

Note.-The values listed are the probabilities of the H $\beta$ FWHM falling within a particular bin, given an input MgII FWHM. All velocities are in log km $\mathrm{s}^{-1}$, and all 
TABLE 2

Statistics of Eddington Ratio Distributions for SDSS

\begin{tabular}{|c|c|c|c|c|c|c|c|c|c|c|}
\hline \multirow[b]{2}{*}{$z_{\text {bin }}$} & \multirow[b]{2}{*}{$\log L_{\text {bin }}$} & \multirow[b]{2}{*}{$N$} & \multicolumn{4}{|l|}{ Raw } & \multicolumn{4}{|c|}{ Mg II Replaced } \\
\hline & & & $\mu$ & $\sigma$ & $S_{k}$ & $A_{k}$ & $\mu$ & $\sigma$ & $S_{k}$ & $A_{k}$ \\
\hline $0.3-1.2$ & $<46$ & 6187 & -0.97 & 0.33 & -0.48 & 4.27 & -0.98 & 0.39 & -0.33 & 3.30 \\
\hline $1.2-2.2$ & $<46$ & 15 & -1.03 & 0.40 & 0.55 & 2.07 & -0.92 & 0.44 & 0.05 & 2.17 \\
\hline $0.3-1.2$ & $46-46.5$ & 5233 & -0.77 & 0.25 & -0.24 & 3.39 & -0.79 & 0.36 & -0.04 & 2.95 \\
\hline $1.2-2.2$ & $46-46.5$ & 5360 & -0.76 & 0.24 & -0.02 & 3.51 & -0.72 & 0.35 & 0.05 & 2.82 \\
\hline $0.3-1.2$ & $>46.5$ & 789 & -0.61 & 0.22 & -0.47 & 3.17 & -0.61 & 0.34 & -0.06 & 2.92 \\
\hline $1.2-2.2$ & $>46.5$ & 9860 & -0.65 & 0.25 & 0.06 & 3.73 & -0.65 & 0.34 & 0.24 & 3.27 \\
\hline
\end{tabular}

Note. - For each bin in redshift $z_{\text {bin }}$ and luminosity $L_{\mathrm{bin}}$, the table lists: $N$, the number of AGNs; $\mu$, the mean logarithm of the Eddington ratio; $\sigma$, the dispersion in $\log \left(L_{\mathrm{bol}} / L_{\mathrm{Edd}}\right)$; $S_{k}$, the skewness; and $A_{k}$, the kurtosis. 


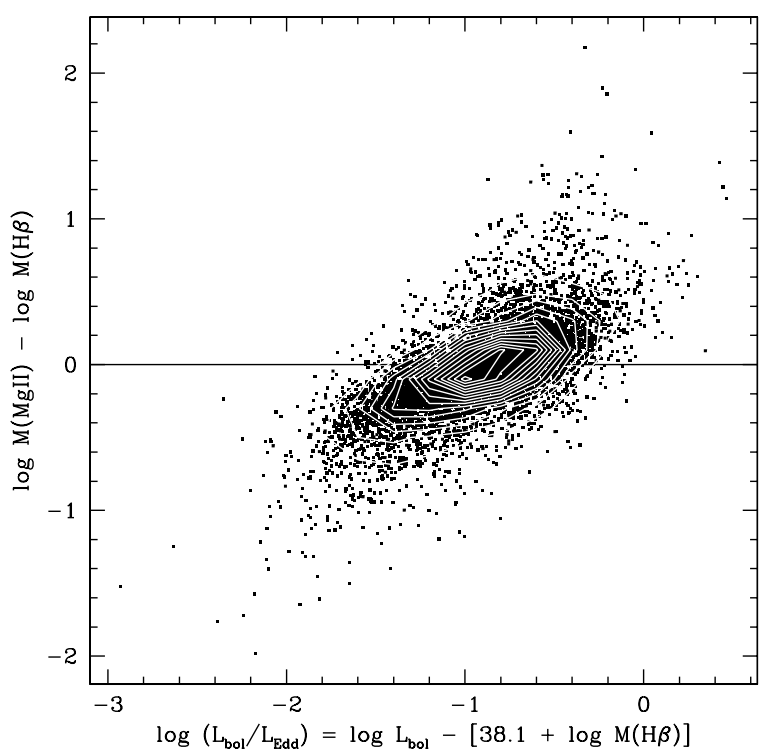

Fig. 1.- Difference in $\mathrm{H} \beta$ and $\mathrm{Mg}$ II estimates of $\log M_{\mathrm{BH}}$ as a function of the $\log$ of the Eddington ratio. The Eddington ratio is calculated using the $\mathrm{H} \beta$ mass estimate. White contours are linearly spaced at intervals of 50 AGNs per $0.2 \times 0.2$-dex bin. The BH mass estimates from Mg II are correlated with the Eddington ratio such that the mass is underpredicted at low Eddington ratio and overpredicted at high Eddington ratio.

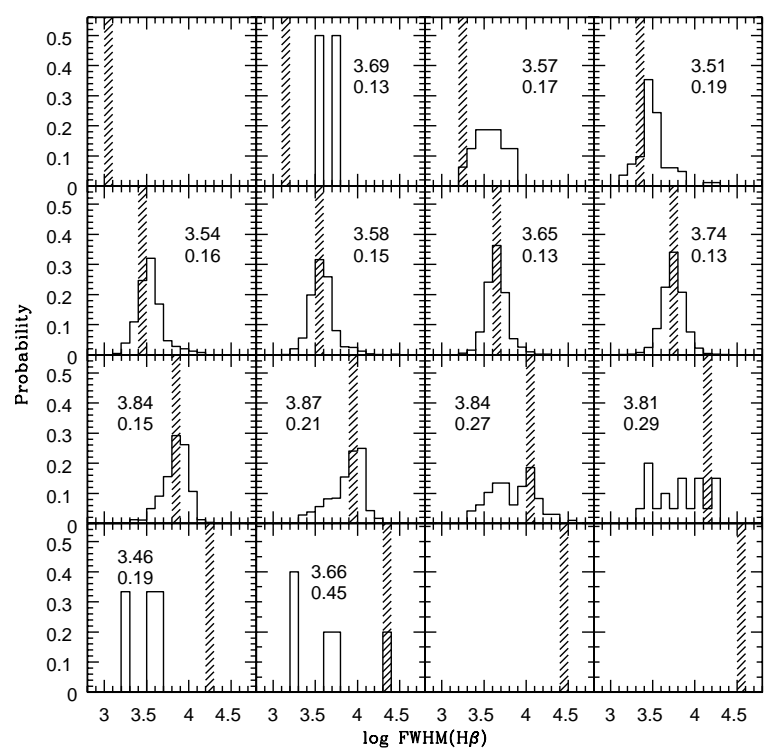

Fig. 2.- Distribution of $\mathrm{H} \beta$ FWHMs for objects having $\mathrm{Mg}$ II FWHM within the 0.1 dex-wide band shown as the crosshatched bar in each panel. The mean and standard deviation in $\log \operatorname{FWHM}(\mathrm{H} \beta)$ is also indicated for each subsample. Despite the overall $\mathrm{H} \beta$ and $\mathrm{Mg}$ II FWHM distributions having similar mean values, the total dispersion in log FWHM is 0.18 dex for the former and only 0.12 dex for the latter, demonstrating that $\mathrm{Mg}$ II fails to reflect the full range of line widths (and hence $\mathrm{BH}$ masses). 


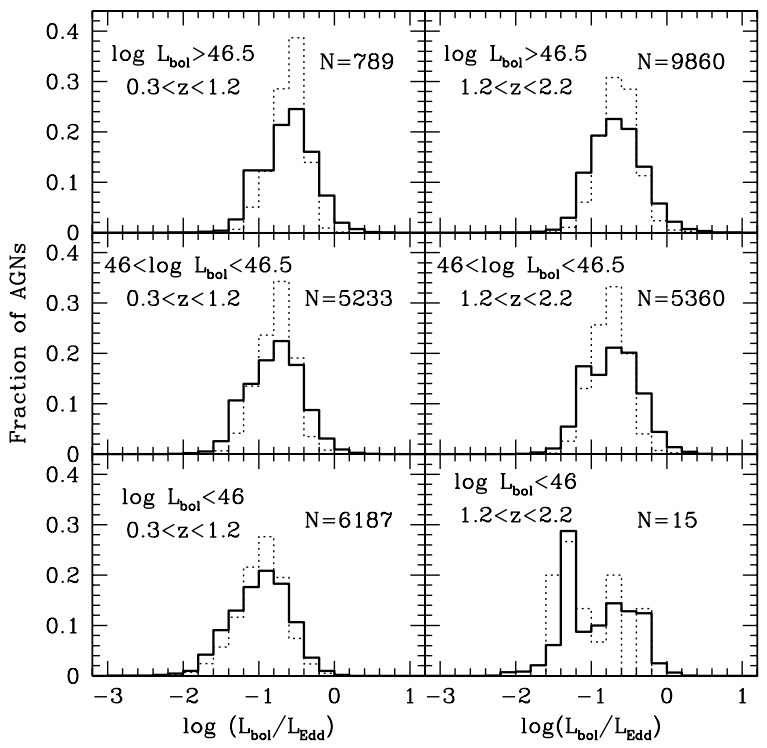

Fig. 3.- Distribution of Eddington ratios in bins of bolometric luminosity and redshift, for the redshift range in which $\mathrm{Mg}$ II estimates of the $\mathrm{BH}$ mass contribute to the distribution. Dotted histograms employ the raw mass estimates from all three emission lines. Solid histograms show the result after replacing each Mg II-based mass with the corresponding probability distribution we derived from $\mathrm{H} \beta$. Bolometric luminosity, redshift, and the number of AGNs are shown in each panel.

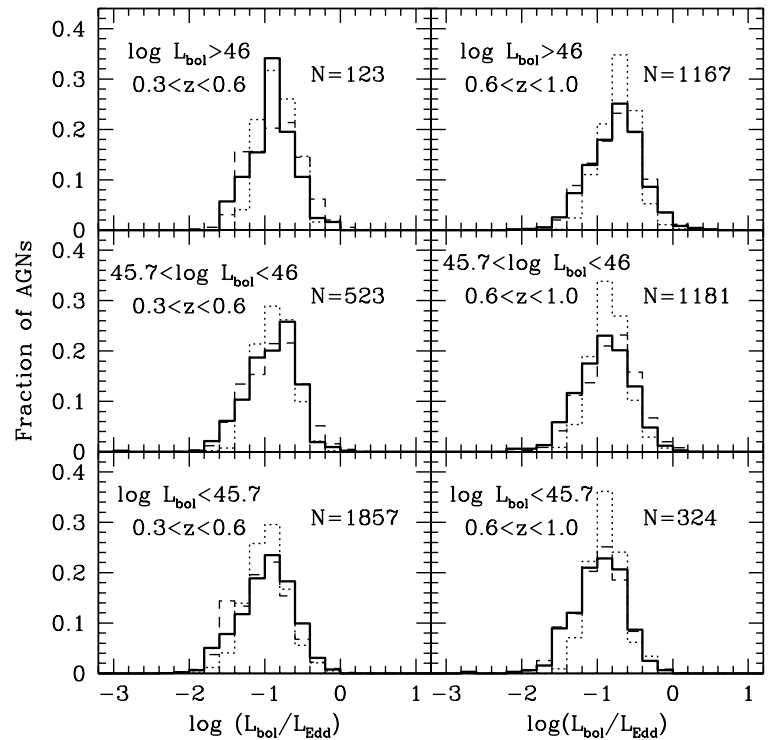

Fig. 4.- Distribution of Eddington ratios in bins of bolometric luminosity and redshift, for objects where both $\mathrm{H} \beta$ and Mg II masses were available. Solid histograms show the distributions for the $\mathrm{H} \beta$ derived masses, dashed histograms show the resultant distributions when our procedure is applied to the Mg II masses, and the dotted histograms show the raw $\mathrm{Mg}$ II estimates. The similarity of the $\mathrm{H} \beta$ (solid) and corrected (dashed) distributions demonstrates that our method of correcting the Mg II masses correctly recovers the true $\mathrm{H} \beta$ Eddington ratio distribution. Bolometric luminosity, redshift, and the number of AGNs are shown in each panel. 